\title{
The Use of Data across Countries: Development and Application of a Data Use Framework
}

\author{
Kim Schildkamp ${ }^{1}$, Louisa Karbautzki ${ }^{2}$, Andreas Breiter ${ }^{2}$, \\ Małgorzata Marciniak ${ }^{3}$, and David Ronka ${ }^{4}$ \\ ${ }^{1}$ University of Twente, The Netherlands \\ k.schildkamp@utwente.nl \\ ${ }^{2}$ Institute for Information Management, University of Bremen, Germany \\ \{karbautzki, abreiter\}@ifib.de \\ ${ }^{3}$ Public Consulting Group, Poland \\ mmarciniak@pcgeu.com \\ ${ }^{4}$ Public Consulting Group, USA \\ dronka@pcgus.com
}

\begin{abstract}
Promising evidence exists that data-based decision-making can result in improvements in student achievement [1], but studies, e.g. [2], show that many schools do not use data properly. Support in the use of data is urgently needed. This chapter focuses on the design of a professional development course in the use of data. In the first phase of the project, case studies were conducted in five participating countries (England, Germany, The Netherlands, Poland and Lithuania) to develop a common data use framework. In the next phase, in two schools in each of the countries, a data use needs assessment was conducted using a survey. Finally, a professional development course was developed and implemented. Results of each of the phases are discussed in this paper.
\end{abstract}

Keywords: Data-based decision making, school improvement, professional development.

\section{Introduction and Theoretical Framework}

Schools internationally are held more and more accountable for the education they provide. This requires them to use data as a basis for their decisions. Promising evidence exists that data-based decision-making can result in improvements in student achievement [1], but studies [e.g. 3] show that many schools do not use data properly. Support in the use of data is urgently needed. This paper focuses on the design of a professional development course in the use of data. The project described here consisted of five phases: case studies; survey administration; course development; course implementation; and course evaluation. This paper focuses mainly on phases 1 to 3 .

Firstly, case studies were conducted to answer the following research questions:

1. For which purposes do school leaders and teachers use data?

2. Which factors influence the use of data?

D. Passey, A. Breiter, and A. Visscher (Eds.): ITEM 2012, IFIP AICT 400, pp. 27-38, 2013.

(C) IFIP International Federation for Information Processing 2013 
The main objective of this phase of the project was to come up with a common data use framework. Since a generally accepted "data use framework" is missing, we developed our own framework based on our literature review, as well as interviews with schools in five European Union (EU) countries and the analysis of policy documents (for an overview of the literature used see [4]). Versions of this framework were published in [3] and [2].

The theoretical framework hypothesizes that several variables with regard to organizational, data and user characteristics influence the use of data. The policy context (such as pressure for achievement) also influences data use within schools. Teachers and school leaders can use data, such as assessment and survey data, for different purposes: for instructional purposes; for supporting conversations with parents, students, fellow teachers, and fellow administrators; for shaping professional development; for encouraging self-directed learning by giving the data to students; for policy development and planning; for meeting accountability demands or complying with regulations; for legitimizing existing or enacted programs; for motivating students and staff; and for decisions related to personnel (e.g. evaluating team performance and determining and refining topics for professional development).

The use of data may lead to an effect on teacher, school leader, and student learning. Teacher and school leader learning is defined in this study as changes in attitude (e.g. towards data use), knowledge (e.g. pedagogic knowledge) and behavior (instructional or leadership strategies) [5]. For example, a teacher who is not satisfied with certain assessment results may decide to analyze the test results more critically. Based on these data he may come to the conclusion that he should make changes in his instruction. As a result, he may start using different instructional strategies (teacher learning: behavior). Data on the next test results can tell him whether or not his changes were successful in terms of whether they led to higher student achievement results (student learning) [6]. However, data use may also have unintended effects, such as stress and de-motivation among school staff as data may give the (surface) impression that they are performing poorly in some aspect of their practice.

\section{Method}

In the five countries participating in this project (England, Germany, The Netherlands, Poland and Lithuania) case studies were conducted. For the case studies, interviews were held with teachers and (assistant) school leaders. We interviewed respondents with regard to which purposes school leaders and teachers use data, and which variables promote and hinder the use of data. In each country at least two schools were included in the interviews. In Germany, 6 teachers and 6 assistant school leaders of two schools were interviewed. In the Netherlands, 11 teachers and 21 assistant school leaders of six schools were interviewed. In Lithuania, 15 assistant school leaders of two schools were interviewed. In Poland, 11 teachers and 2 assistant school leaders of two schools were interviewed. In England, 6 teachers and 8 assistant school leaders 
of four schools were interviewed. Documents (e.g. policy plans, literature, and Organisation for Economic Development (OECD) reports) were collected to describe the educational policy (related to data use) in each of the countries.

Based on the data use framework that was developed from the case studies, we conducted a survey study to diagnose data use practices within two schools in each of the countries (e.g. the partner schools) and their current capacity to use data effectively. Participants were asked to rate 78 data use-related items [4] by their accuracy or frequency. The survey was administered prior to and after the data use course. In 2011, 398 teachers from 10 schools in 5 countries completed the survey. In 2012, 228 responses from 7 schools in 4 countries were collected.

The collected data were analyzed in two ways. Firstly, school reports with diagrams, means and standard deviation values for every survey item were created to give feedback to the pilot schools. Secondly, the data were analyzed in a crosscountry exploratory factor analysis by specification search [7] to determine which areas of data use influence one another. The factors derived from the survey items were: (1) data accessibility, (2) data quality, (3) user attitudes, (4) user skills, (5) school leadership, (6) school cooperation, (7) school vision and norms, (8) school training and support, (9) using data for accountability, (10) using data for school development, and (11) using data for instructional development.

The results of the case studies and data use survey, combined with an analysis of existing data use courses $[6,8,9]$, informed the development of a data use professional development course which was designed to address the documented lack of capacity of school leaders and staff to effectively use data to improve student outcomes. The goals for the course were developed within a 5-stage data inquiry model that served as an inquiry framework to support participants as they learned how to use data for problem solving and decision-making in their schools. Eleven professional development modules were developed to meet the goals within each phase of the data inquiry model.

\section{Results - Case Studies}

\subsection{Policy Contexts}

Five different countries participated in the project with different policy contexts. Germany has 16 different states, and each state is responsible for providing education. Within the states, schools are centrally organized, and very limited autonomy exists for schools $[10,11]$. Germany has a standard curriculum or partly standardized curriculum that is required, as well as mandatory national examinations and assessments [11]. Internal evaluations are not compulsory, but school boards and other organizations offer tools and support. Types of data that are available differ by state: all have student achievement results; some have inspection and self-evaluation results. Little to no support exists for schools in the use of data.

England works with published national data sets (League tables). Schools in England have a significant amount of autonomy $[10,11]$. England does have a standard 
curriculum, mandatory national assessments are required in state schools, but national examinations are optional but rarely not taken [11]. Schools are inspected by Ofsted, who provide schools with inspection reports. Internal evaluations, using lesson observation, perception questionnaires, attainment and achievement data, are highly recommended. External inspections from external evaluation agencies are optional. There is an expectation that teachers will undertake continuous professional development activities and approximately 5 teaching days (out of 195) are allocated to this activity. Also, England has a national student database, and achievement and attainment tables, which makes important information available in a systematic and largely accessible manner.

In Lithuania, schools are evaluated both externally and internally. External evaluations are carried out by the National Agency for School Assessment. Internal evaluations are required as well. Schools can use the internal audit methodology developed by the National Agency for Internal Evaluation or use their own system. Internal evaluations are carried out by the school administration in cooperation with teachers.

An important legislative act for Polish education is the Pedagogical Supervision Act, passed in 2009. The act states that all schools in Poland must be externally evaluated by education authorities. The Ministry of National Education provides curriculum standards, districts and municipalities control administration and financing. School directors have autonomy as far as the decision making around hiring teachers, approving programs and textbooks, conducting internal evaluations. Poland has mandatory national examinations and assessments. A lot of data are available; however these are mostly (value added) achievement data. Schools have electronic data systems in place.

In the Netherlands, schools have significant autonomy. Similar to England, almost all decisions are made at the level of the school $[10,11]$. The Netherlands does not have a standard curriculum or partially standard curriculum that is required; it does have mandatory national examinations, but no national assessments [11]. However, schools are held accountable for their functioning by the Dutch Inspectorate. As schools are responsible for the quality of education they provide, they have to conduct a school self-evaluation to check their quality and improve if necessary. Different consultancy organizations offer data use trainings, but participation is up to schools.

\subsection{Data Available and Data Use}

The results of the case studies show that in both German schools a lot of data are collected, but are not systematically used. School leaders mainly use data for administrative purposes. Teachers use data to monitor progress of students and to determine the need for individual student support or instructional changes. Probably partly due to the English context, a wide range of different types of data are used for different purposes by the four schools included in this study, ranging from accountability purposes to school improvement purposes. The results of the interviews in the two Lithuanian schools show that schools would like to use data more extensively than they do, currently mostly using different types of data for monitoring purposes. Data are used for a wide range of purposes in Poland, including monitoring purposes and 
improvement purposes, but this only happened in small pockets. The results of the interviews in six Dutch schools show that, although a wide range of input, process and output data are available, the use of data is rather limited. Only in two of the schools were data really being used by teachers and school leaders for school improvement purposes (for more detailed results see [4]).

\subsection{Influencing Factors}

Firstly, data and data system characteristics can influence the use of data. In Germany, Lithuania, and the Netherlands access and availability of data were found to be a problem sometimes. Especially in Germany, respondents indicated that this was a problem in their schools. One of the respondents stated "schools have only limited access to data and no real autonomy". Often data are not fed back (e.g. availability) to the schools and the decisions based on these data are not transparent. Moreover, the data collection for the national learning performance measurements is carried out within the school and is very error-prone, resulting in low quality data. Some respondents of the UK, Lithuania and the Netherlands also indicated that there were problems with the quality of the data in terms of, for example, accuracy, but especially with regard to timeliness. Lithuanian respondents indicated that external and internal evaluation did result in usable, relevant, reliable and accurate data. Respondents in Poland all indicated that the data available were perceived as reliable, valid, and accurate. England has the most sophisticated data systems and data tools available. For example, English schools have access to a national student database, and achievement and attainment tables, which makes a lot of needed information available in a systematic and largely accessible manner. Overall schools have access to data systems, a wide range of data and also have tools available to analyze and use data. In contrast, in Germany a problem existed with regard to interoperability between the different data sets. Hence, the relation between different data could not be analyzed. Moreover, problems existed with regard to the ICT infrastructure. Finally, respondents in the Netherlands complained that there was too much data out there and that "data are not always accessible, partly because there are too much data available".

Secondly, organizational characteristics can influence data use. A lack of time and money was found to impede data use in schools. Several respondents complained about a lack of time to use data (Germany, UK, The Netherlands). Collaboration was found to be an important promoting factor of data use. Collaboration around the use of data was not common practice in Germany. Teachers in England, Lithuania, Poland and the Netherlands did collaborate around the use of data (to some extent). For example, in Poland, in one of the schools, teachers collaborated usually in subject specific team meetings, where student outcome data was analyzed, sometimes at the request of the school leader. The school leader coordinated and supported the work of the teams in one of the schools. A lack of training can impede effective data use. In England, different types of training as well as data tools were available. Lithuanian, Dutch and Polish respondents indicated needing training in the use of data. Also, the availability of a data expert, as was found in England and the Netherlands, can promote data use. Lithuanian respondents indicated a need for a data expert. Having a 
clear vision, norms and measurable goals can promote data use. English and Dutch respondents mentioned that the school had established a clear vision with regard to data use and clear and measurable goals. Lithuanian respondents also mentioned this, as well as using data to monitor the implementation of this vision and these goals.

Finally, user characteristics can either promote or hinder data use. In Germany and Lithuania, respondents indicated a lack of data analyses and data use skills. In England, some teachers indicated having the necessary knowledge and skills; others indicated they needed further professional development. Respondents in both Polish schools believed in the use of data. Moreover, in one school, teachers had the knowledge and skills needed to work with data, as these teachers were certified examiners. In the other school, teachers indicated lacking the skills to use value added data. Moreover, in the Netherlands, school leader support and a belief in the use of data were found to be important. An external locus of control present in two of the schools may have hindered data use as these teachers stated that "assessment results are different each year, depending on whether you have good or not so good students" (for more detailed results see [4]).

Based on the results described above, we developed the data use framework displayed in Figure 1. In this framework, policy influences the enablers and barriers to data use, data-driven decision making, and stakeholder and student learning. Different aspects of a country's policy may be of influence. Firstly, characteristics of the accountability system may play an important role. For example, the presence of an inspectorate (such as in England and in the Netherlands) and other forms of external evaluation (such as in Poland, Lithuania) may influence data use. Schools may perceive these evaluations as a form of pressure. Another form of pressure that is put on schools is the public presentation of school performance, such as in England (in League tables), in the Netherlands (online and in rankings that appear in newspapers and journals) and Lithuania (online). Moreover, schools in some countries, such as Lithuania, the Netherlands and England, are expected to engage in school selfevaluations, leading to additional data schools can use. Also, the amount of autonomy schools have in decision making can affect data use. In England and the Netherlands, schools have a lot of autonomy, and they can make almost all decisions (with regard to the curriculum, instruction, personnel and resources) themselves. In Germany, schools have a lot less autonomy. Furthermore, a policy context influences the types of data that are available. Some countries work with national standardized assessments (Germany and Poland) and/or national standardized examinations (Germany, Poland and the Netherlands). Finally, if a country offers training (and sometimes a reduction in teaching hours as a consequence of taking the training) this can influence the extent to which school staff are able to engage in effective data use.

As displayed in Figure 1, different enablers and barriers influence data-driven decision making. Firstly, characteristics of the organization influence data-driven decision making. The following organization variables were found to influence data-driven decision making in the different countries (e.g. in some countries the lack of these variables hindered effective data-driven decision making, while the presence of these variables promotes effective data-driven decision making): 
- Structured time is set aside to use data and structured processes for data use exist within the school (G, N, L, E, P) (Note: G, N, L, E, P refer respectively to German, Netherlands, Lithuanian, English and Polish schools).

- The availability of an data expert who can provide the needed data in a timely matter, as well as assist in analyzing, interpreting, and using data (G, N, L, E).

- The availability of training: professional development in accessing, analyzing, interpreting and using data $(\mathrm{G}, \mathrm{N}, \mathrm{L}, \mathrm{E})$.

- Teacher collaboration: teachers collaborate around the analysis, interpretation and use of data, in for example subject matter teams, grade level teams or data teams (G, N, L, P, E).

- Vision, norms and goals for data use: the school has clear goals and visions, and data can be used to monitor the extent to which the school is reaching these as well as to come up with measures to improve, if necessary. Moreover, the school also expects school staff to use data on a regular basis and specific norms and goals with regard to data use exists $(\mathrm{G}, \mathrm{N}, \mathrm{L}, \mathrm{E})$.

- The school leader actively supports, encourages and facilitates data use (for example, by structuring time) $(\mathrm{N}, \mathrm{P})$.
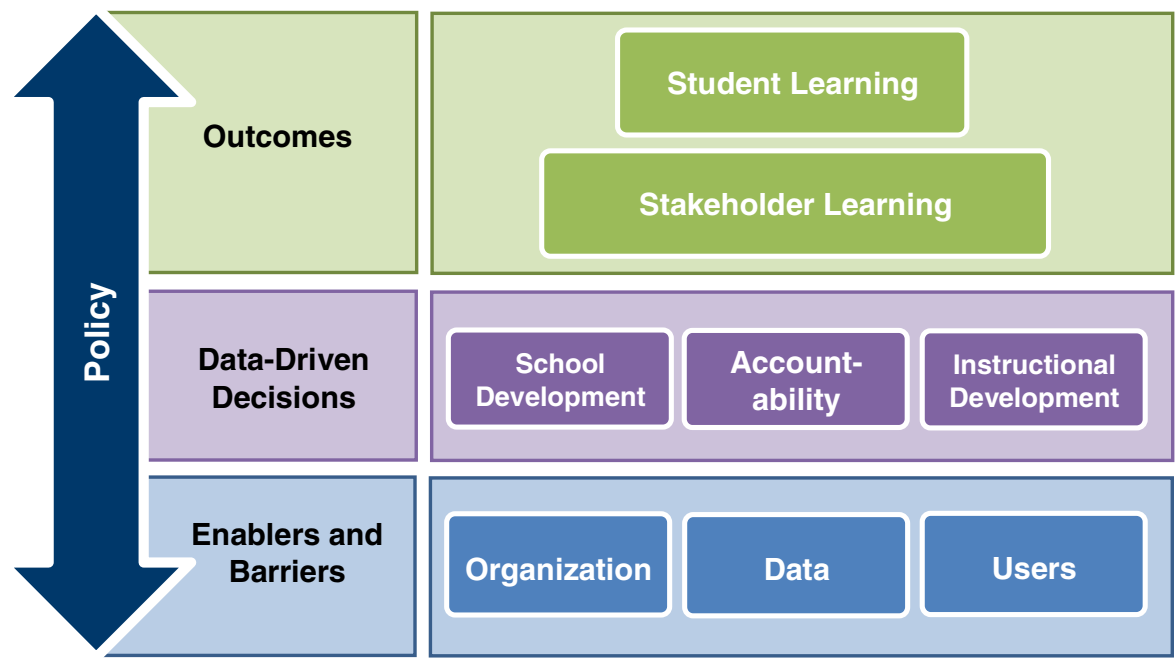

Fig. 1. A data use framework

Secondly, characteristics of the data influence use. Specifically, the following variables were found to play a role:

- Accessibility and availability of data and information logistics, for example through information management and other data systems. School staff should be able to find the data they need easily and timely. Data should be aligned, and school staff should not have to look into three different systems to obtain the types of data they need (G, N, L, P, E).

- The quality of data: schools need timely, accurate, valid, relevant, reliable data, which coincides with their needs $(G, N, L, P, E)$. 
- Tools, which support data analyses, interpretation and use (e.g. which can aggregate data, can calculate attainment and progress, adjusted to socio-economic status etc.) $(\mathrm{G}, \mathrm{N}, \mathrm{E})$.

Thirdly, user characteristics influence data use. The following variables were found to play a role in the different countries:

- Attitude toward data: It is important that school staff believe in the use of data, that they think it is necessary to use data to improve their practice, and that they are motivated to use data $(\mathrm{G}, \mathrm{N}, \mathrm{L}, \mathrm{P}, \mathrm{E})$.

- School staff need knowledge and skills to collect, analyze, interpret and use data (G, N, L, P, E).

The different enablers (if these variables are present) and barriers (absence of these variables) influence the extent to which data are used to base decisions on. We distinguish between three different types of data-driven decision making (although these sometimes overlap). Firstly, the use of data can be used for school development purposes. In the case studies described above the following school development purposes were mentioned:

- Policy development and school improvement planning based on areas of need and strong aspects (N, E, G, L, P).

- Teacher development (G, N, L, P, E).

- Grouping of students and placing students at school level (G, P, E).

- Monitoring the implementation of the school's goals and, if necessary, (re)defining aims and objectives/setting new targets $(\mathrm{L}, \mathrm{E})$.

- Motivating staff $(\mathrm{E})$.

Data can also be used for accountability purposes. The following accountability purposes were identified in the case studies:

- Public relations, to show the outside world how good the school is doing $(\mathrm{G}, \mathrm{N})$.

- Communication with parents (e.g. schools are accountable to parents) (G, L, P, E).

- Communication with other schools (L).

- To meet accountability demands (for example, self-evaluation results are used as a basis in external evaluations) (N, E, P).

Thirdly, data can be used for instructional development, such as:

- Monitoring progress of students (G, N, L, P, E).

- Adjusting instruction (e.g. adapting instruction towards the needs of students, grouping students differently, determining the content of instruction, giving students feedback, providing students with additional time etc.) (G, N, P, E).

- Curriculum development (P, E).

- Motivating and rewarding students (E).

If data are used for these different purposes, this may lead to stakeholder (e.g. teachers, school leaders, parents) learning. For example, a teacher might decide to make instructional changes based on data (data-driven decision). This leads to improved instruction by the teacher (outcome: teacher stakeholder learning). Stakeholder learning in turn may lead to student learning (e.g. inquiry of students into their own learning and improved student achievement). 


\section{$4 \quad$ Results Survey}

Based on the data use framework described above, a data use survey was developed and administered to ten schools in the five countries. The cross-country survey analysis shows that the intention of the Comenius project is targeting an area that is both valued as of high importance by the survey respondents and at the same time significantly underdeveloped. More than 50 per cent of the respondents (strongly) agreed that "it is important to use data to diagnose individual student learning needs" and that "data can offer information about students that was not already known". Moreover, more than half of the respondents stated that their "principal or assistant principal(s) encourage data use as a way to support effective teaching". And finally, the cooperation level seems also promising: Most "would like to collaborate more with other educators about using data", their school "effectively communicates school improvement goals" to them, and the respondents "share and discuss student performance data with students, parents and other teachers".

A correlation analysis further showed that all factors derived from the survey items influence each other. The factor analysis revealed which of them describe the current status of data use. The structural equation models in Figure 2 visualize these results for the cross-country sample in 2011 and 2012.
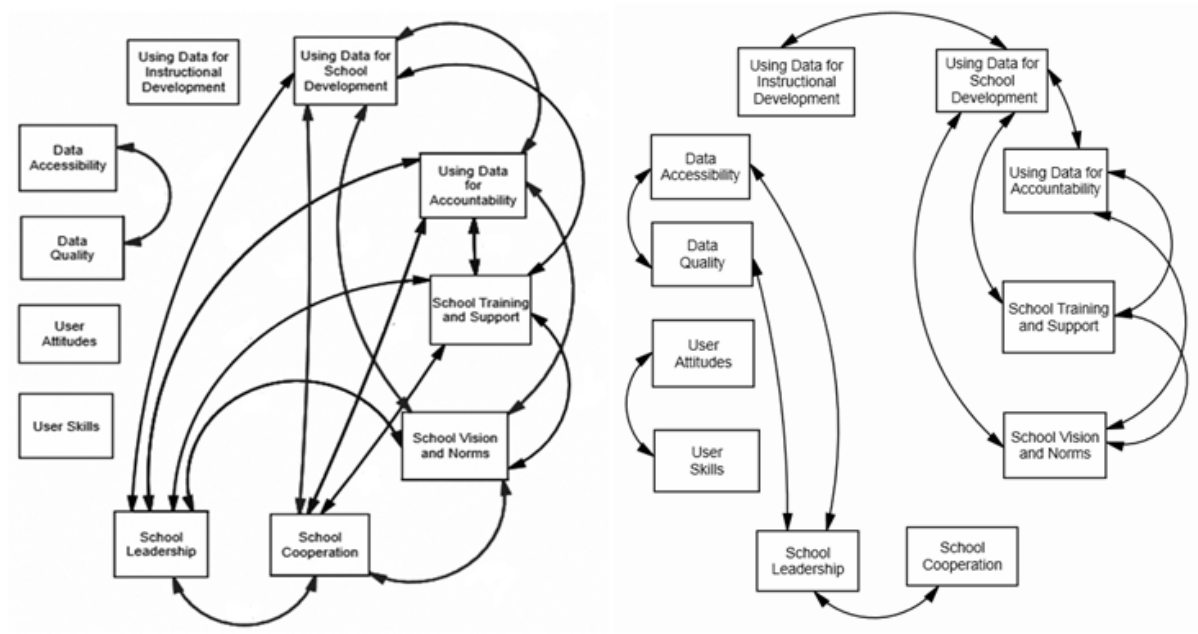

Fig. 2. Cross-country models with correlations between factors 2011 (left) and 2012 (right)

A look at the 2011 model revealed important insights for the further development of the project. Certain factors are linked to others very often and others are not linked at all. School Leadership, for example, is linked to five other factors. Also, most of these five are interlinked. This means that these factors have the highest influence on each other and thus on aspects of data use in all countries. This reflects the results of nearly all empirical studies on school improvement in which the role of the school leader is regarded as a necessary condition for change $[12,13]$. The factors that are 
not linked at all in the 2011 model are User Skills, User Attitudes and Using Data for Instructional Development, which are the key elements in the goals of the Comenius project. At this stage, the skills and mind-set of EU teachers in this cross-country sample to use data for instructional development are significantly underdeveloped. In 2012, this changed. Using Data for Instructional Development plays a more active role and User Attitudes and Skills change together. Also, School Leadership is now connected to factors outside the school realm and has important correlations with Data Accessibility and Quality.

\section{Characteristics of the Data Use Course}

The results of case studies and survey, combined with the analysis of existing data use courses, informed the development of the curriculum and the content of the data use course described in this paper. It consists of 11 obligatory modules which are organized according to the 5 stages of a data inquiry model: preparation; discovery; diagnosis; doing, and evaluation (see Figure 3). The course was designed to guide teams of teachers and school leaders (professional learning communities (PLCs)) through a structured and iterative application of the 5-stage inquiry model, relying heavily on data analysis and collaboration protocols, templates and tools, and authentic learning activities to build the necessary technical, analytical, and collaboration skills.

Modules 1 and 2 (Getting Started and Data Literacy) are Preparation modules that build the capacity of the PLC to work collaboratively with data. Modules 3 to 11 guide the PLC through each stage of the data-driven inquiry model: Discovery (Identifying a Problem, Evaluating and Analyzing Data); Diagnosis (Hypothesizing and Analyzing Root Causes); Doing (Brainstorming Initiatives, Developing Action Plans and Monitoring Implementation); and Evaluation in which they reflect upon the initiatives they implemented throughout the course. The course helps build the capacity of the school-based teams to not only engage in their own inquiry, but to serve as catalysts for whole-school data-driven decision making (for more information on the data use course see: www.datauseproject.eu).

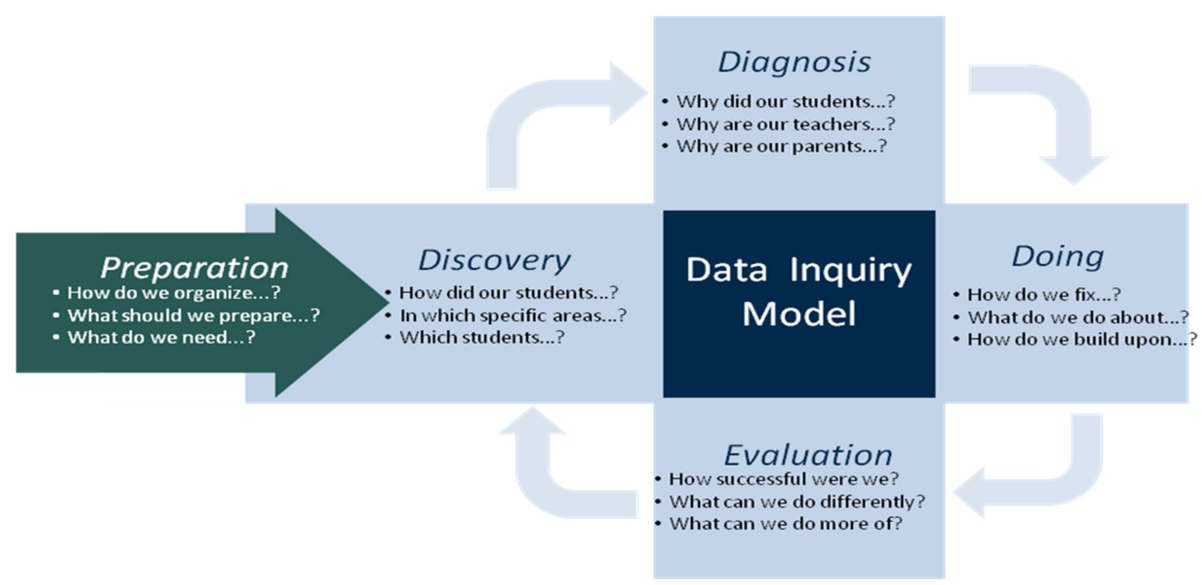

Fig. 3. The Data-Driven Inquiry Model 


\section{Conclusion and Discussion}

Before coming to conclusions, we have to discuss the limitations of this study. First of all, the schools that participated are no means a representative sample. We want to emphasize here that the goal of this part of the project was not to make firm generalizations, but to gain more insights into the use of data in different schools. The data were collected by interviewing teachers and school leaders and by administering surveys in only a few schools per country. Teachers' and school leaders' self-perceptions were used to study their use of data. We used triangulation (e.g. interviews, documents and surveys) and we checked the comments made by the respondents by asking for more details and by asking for examples. Still, the data may produce a slightly colored or biased picture of the actual use of data within schools. Lastly, we conducted some of our analyses across countries and based on combined school leader and teacher data. This might have led to the loss of certain nuances, and of course we do acknowledge that there are differences between the use of data by school leaders and teachers [see 3] and between the use of data between but also within countries and within schools. However, as the results are in line with the results of several other data use studies, we do feel confident that the results of this study present an accurate picture of the current use of data in schools in different countries.

Based on the case studies, a data use framework was developed, which informed the development of the data use survey. The survey analysis was an important first step in the process of establishing a school-wide culture of data use. It captured the schools' areas of strengths as well as areas for improvement under key data use categories. According to the survey results, schools in Poland, Lithuania and England took the lead in the practice of data use while the schools from The Netherlands and Germany show lower levels of activities in many areas. However, respondents in all countries indicted a need for (further) provision of training and support in the use of data, which the data use course we developed tried to provide.

All ten schools in the five participating countries implemented the data use course and followed it for a period of one school year. Although it is not within the scope of this paper to discuss the exact evaluation results, we do want to highlight some of the findings. The evaluations showed that among the major challenges in using data in schools are: the tendency of teachers to take action quickly (and not always based on data) and to think that the action will have an immediate impact; the length of the inquiry process; the need for whole-faculty involvement; the amount of time and effort required for the inquiry process; and resistance from teachers who do not fully understand how data can help them improve teaching and learning.

PLC members also stated that those who engage in a structured data-driven inquiry process should be aware that "data use" requires not only the analysis of qualitative and quantitative data, but also a commitment to an improvement process that requires time and effort. Collaboration requires a large level of involvement from all of the team members. Engaging successfully in the process of data-driven improvement also requires that all faculty members are willing to reflect upon and share their instructional practice and productively discuss the challenges in the school. These are not cultural norms in many schools, but they are absolutely critical to the process of 
continuous improvement. Finally, the PLC members pointed out that using data in their school allowed them to see things that were not immediately evident, and that it has been worthwhile to collect data, analyze it, and collectively plan for concrete school improvements that will ultimately help prepare their students for academic success.

Acknowledgements. This paper includes parts of the report "Comparative analyses data use in Germany, The Netherlands, Lithuania, Poland and England" on the workpackage lead by Dr Kim Schildkamp, University of Twente and "Survey Data Analysis" on the workpackage lead by ifib Institute for Information Management, Bremen GmbH, authored by PCG Polska (PL), Specialist Schools and Academies Trust (UK), Modern Didactics Center (LT), University of Twente (NL), and the Institute for Information Management, Bremen $\mathrm{GmbH}(\mathrm{GE})$. All rights are reserved to DATAUSE project partners. The DATAUSE project has been funded with support from the European Commission. This paper reflects the views of the authors only, and the Commission cannot be held responsible for any use which may be made of the information contained herein.

\section{References}

1. Carlson, D., Borman, G., Robinson, M.: A multistate district-level cluster randomized trial of the impact of data-driven reform on reading and mathematics achievement. Education and Evaluation and Policy Analysis 33(3), 378-398 (2011)

2. Schildkamp, K., Lai, M.K., Earl, L.: Data-driven decision making around the world: Challenges and opportunities. Springer, Dordrecht (2012)

3. Schildkamp, K., Kuiper, W.: Data informed curriculum reform: Which data, what purposes, and promoting and hindering factors. Teaching and Teacher Education 26, 482-496 (2010)

4. Comenius Project Using Data for Improving School and Student Performance, Comparative analyses data use in Germany, The Netherlands, Lithuania, Poland and The United Kingdom and DATA USE Survey (2011),

http: / /www. datauseproject. eu/home/documents

5. Guskey, T.R.: Attitude and perceptual change in teachers. International Journal of Educational Research 13(4), 439-453 (1989)

6. Boudett, K.P., Steele, J.L.: Data wise in action. Stories of schools using data to improve teaching and learning. Harvard Education Press, Cambridge (2007)

7. Anderson, T.W.: An introduction to multivariate statistical analysis, 3rd edn. Wiley, Hoboken (2003)

8. Ronka, R., Geier, R., Marciniak, M.: How a focus on data quality, capacity, and culture supports data-driven action to improve student outcomes. PCG, Boston (2010)

9. Schildkamp, K., Handelzalts, A., Poortman, C.L.: Data teams for school improvement. Paper Presented at the AERA Conference, Vancouver, Canada (2012)

10. OECD: Education at a glance 2008: OECD indicators. Organisation for Economic Cooperation and Development, Paris (2008)

11. OECD: Education at a glance 2010: OECD indicators. Organisation for Economic Cooperation and Development, Paris (2010)

12. Fullan, M.G.: Leading in a Culture of Change. Jossey-Bass, San Francisco (2011)

13. Hattie, J.A.C.: Visible learning: a synthesis of over 800 meta-analyses relating to achievement. Routledge, London (2009) 